\title{
A Domain Decomposition Based Parallel Inexact Newton's Method with Subspace Correction for Incompressible Navier-Stokes Equations ${ }^{\star}$
}

\author{
Xiao-Chuan $\mathrm{Cai}^{1}$ and Xuefeng $\mathrm{Li}^{2}$ \\ 1 Department of Computer Science, University of Colorado at Boulder \\ Boulder, CO 80309 \\ cai@cs.colorado.edu \\ 2 Department of Mathematics, \\ Loyola University New Orleans, New Orleans, LA 70118 \\ li@loyno.edu
}

\begin{abstract}
There are two major types of approaches for solving the incompressible Navier-Stokes equations. One of them is the so-called projection method, in which the velocity field and the pressure field are solved separately. This method is very efficient, but is difficult to be extended to another multi-physics problem when an appropriate splitting is not available. The other approach is the fully coupled method in which the velocity and pressure fields stay together throughout the computation. The coupled approach can be easily extended to other multiphysics problems, but it requires the solution of some rather difficult linear and nonlinear algebraic systems of equations. The paper focuses on a fully coupled domain decomposition based parallel inexact Newton's method with subspace correction for incompressible Navier-Stokes equations at high Reynolds numbers. The discussion is restricted to the velocity-vorticity formulation of the Navier-Stokes equations, but the idea can be generalized to other multi-physics problems.
\end{abstract}

\section{Introduction}

In this paper we develop inexact Newton type methods [6] that use local subdomain corrections for large nonlinear systems of algebraic equations, arising from the discretization of nonlinear partial differential equations. All systems considered in the paper have a common feature - local high nonlinearities. In other words, the nonlinear system may have many equations, but only a small percentage of them are highly nonlinear compared to the rest of the equations. These local high nonlinearities often correspond to boundary or interior layers, or corner singularities 316. Global inexact Newton's methods may be used to solve the system, but often computing time is wasted since all equations are treated equally as if they were all highly nonlinear. We introduce local zeroth- and

\footnotetext{
* The research was supported in part by DOE under DE-FC02-04ER25595, and in part by NSF under grants ACI-0305666, CNS-0420873, CCF-0634894, and CNS-0722023.
}

G. Allen et al. (Eds.): ICCS 2009, Part I, LNCS 5544, pp. 795-803, 2009.

(C) Springer-Verlag Berlin Heidelberg 2009 
first-order Jacobi method to remove the local high nonlinearities and therefore improve the efficiency and the effectiveness of the outer global inexact Newton's method [7,15, which performs well on equations with roughly the same level of nonlinearities.

We provide numerical results to demonstrate its effectiveness as compared to the classical inexact Newton's method. As an example, we show numerically that the method performs well for solving the two-dimensional nonlinear driven cavity flow problem 8]. Using the velocity-vorticity formulation, in terms of velocity $u, v$, and vorticity $\omega$, the driven cavity flow problem on unit square $\Omega=(0,1) \times(0,1)$ is

$$
\left\{\begin{aligned}
-\Delta u-\frac{\partial \omega}{\partial y} & =0 \\
-\Delta v+\frac{\partial \omega}{\partial x} & =0 \\
-\frac{1}{R e} \Delta \omega+u \frac{\partial \omega}{\partial x}+v \frac{\partial \omega}{\partial y} & =0 .
\end{aligned}\right.
$$

Here $R e$ is Reynolds number. The boundary conditions are:

bottom, left and right: $u=v=0$; top: $u=1, v=0$.

The boundary condition on $\omega$ is given by its definition:

$$
\omega(x, y)=-\frac{\partial u}{\partial y}+\frac{\partial v}{\partial x} .
$$

We vary the Reynolds number in the experiments. Using the usual uniform mesh finite difference approximation with the 5-point stencil (upwinding for the convective terms and central differencing for the other terms) we obtain a system of nonlinear equations in the form of

$$
F(u)=0
$$

where $u=\left(u_{1}, \cdots, u_{N}\right)^{T}, F=\left(f_{1}, \cdots, f_{N}\right)^{T}$ and $f_{i}=f_{i}\left(u_{1}, \cdots, u_{N}\right)$. Here $N$ is the total number of unknowns. Classical Newton type algorithms, e.g. 6], do not assume that nonlinearities in functions $f_{1}, \cdots, f_{N}$ are too much different. This is often fine when the number of functions is small, but is not acceptable when $N$ is sufficiently large. Recent experiences show that, in many large scale multiphysics problems, the nonlinearities are far from balanced. In many situations, only a small percentage of the components of $F$ are highly nonlinear. To improve the efficiency of Newton's methods, in this paper, we develop a locally adaptive version of Newton's method which uses some special treatments for the highly nonlinear components of $F$.

For solving large scale problems, parallel processing is a must, and we assume here that domain decomposition [14 is used with a partition of the vector $u$, as well as $F$, into subdomains

$$
u_{\Omega_{1}}, \cdots, u_{\Omega_{p}}, \text { and } F_{\Omega_{1}}, \cdots, F_{\Omega_{p}} \text {. }
$$


With this partition, the nonlinear system takes a block form as follows.

$$
\left\{\begin{array}{l}
F_{1}\left(u_{\Omega_{1}}, \cdots, u_{\Omega_{p}}\right)=0 \\
F_{2}\left(u_{\Omega_{1}}, \cdots, u_{\Omega_{p}}\right)=0 \\
\vdots \\
F_{p}\left(u_{\Omega_{1}}, \cdots, u_{\Omega_{p}}\right)=0
\end{array}\right.
$$

The classical Newton's method goes like

$$
\left(\begin{array}{l}
u_{\Omega_{1}}^{n} \\
\vdots \\
u_{\Omega_{k}}^{n} \\
\vdots \\
u_{\Omega_{p}}^{n}
\end{array}\right)=\left(\begin{array}{l}
u_{\Omega_{1}}^{n-1} \\
\vdots \\
u_{\Omega_{k}}^{n-1} \\
\vdots \\
u_{\Omega_{p}}^{n-1}
\end{array}\right)+J^{-1}\left(\begin{array}{l}
F_{1}\left(u_{\Omega_{1}}^{n-1}, \cdots, u_{\Omega_{k}}^{n-1}, \cdots, u_{\Omega_{p}}^{n-1}\right) \\
\vdots \\
F_{k}\left(u_{\Omega_{1}}^{n-1}, \cdots, u_{\Omega_{k}}^{n-1}, \cdots, u_{\Omega_{p}}^{n-1}\right) \\
\vdots \\
F_{p}\left(u_{\Omega_{1}}^{n-1}, \cdots, u_{\Omega_{k}}^{n-1}, \cdots, u_{\Omega_{p}}^{n-1}\right)
\end{array}\right) .
$$

As one can tell from (5), all functions $F_{i}$ participate in all iterations no matter where the high nonlinearities are.

On the other hand, there is another well-known method called nonlinear Jacobi method [12], and its block form can be presented as follows: Let $u_{\Omega_{1}}^{n}, \cdots, u_{\Omega_{p}}^{n}$ be the current approximate solution on each subdomain, then the new iteration on subdomain $\Omega_{i}, u_{\Omega_{i}}^{n+1}$, is computed by solving

$$
F_{i}\left(u_{\Omega_{1}}^{n}, \cdots, u_{\Omega_{i}}^{n+1}, \cdots, u_{\Omega_{p}}^{n}\right)=0,
$$

using Newton's method on $\Omega_{i}$ with $u_{\Omega_{i}}^{n}$ as the initial guess. These subdomain problems can be solved independently. This nonlinear Jacobi method has the perfect parallelism, and is a local method in the sense that if the output of the subdomain function on $\Omega_{k}$ is more nonlinear than others, then more subdomain Newton iterations are needed only on this particular subdomain. No other subdomain needs to participate in the lengthy iterations, as the global Newton's method does. However, the nonlinear Jacobi method is not being used much in practice because of its slow convergence. The focus of this paper is to combine the non-proliferation properties of Jacobi and the fast convergence of global Newton's methods.

For simplicity, we assume that the high nonlinearity is mainly in the area of $\Omega_{k}$, and as a result, the subdomain residual function $\left\|F_{\Omega_{k}}\right\|_{2}$ is larger than residuals in other subdomains, i.e.,

$$
\left\|F_{\Omega_{k}}\right\|_{2} \geq\left\|F_{\Omega_{i}}\right\|_{2}, \quad i \neq k .
$$

In other words, the subsolution in the $k^{\text {th }}$ subdomain $u_{\Omega_{k}}^{n}$ is not as good as subsolutions in other subdomains. To improve the situation in subdomain $\Omega_{k}$, or say to balance the overall nonlinearity, we could stop the global Newton iteration temporarily and focus on subdomain $\Omega_{k}$ using the following subdomain Newton iterations:

$$
u_{\Omega_{k}}^{n, m}=u_{\Omega_{k}}^{n, m-1}-J_{\Omega_{k}}^{-1} F_{\Omega_{k}}\left(u_{\Omega_{1}}^{n}, \cdots, u_{\Omega_{k}}^{n, m-1}, \cdots, u_{\Omega_{p}}^{n}\right),
$$


with the initial guess $u_{\Omega_{k}}^{n, 0}=u_{\Omega_{k}}^{n}$. Suppose Algorithm (77) converges, then the subdomain Newton iterations will result in

$$
\lim _{m \rightarrow+\infty} u_{\Omega_{k}}^{n, m}=v_{\Omega_{k}}^{n},
$$

that is

$$
v_{\Omega_{k}}^{n}=v_{\Omega_{k}}^{n}-J_{\Omega_{k}}^{-1} F_{\Omega_{k}}\left(u_{\Omega_{1}}^{n}, \cdots, v_{\Omega_{k}}^{n}, \cdots, u_{\Omega_{p}}^{n}\right),
$$

which implies that

$$
F_{\Omega_{k}}\left(u_{\Omega_{1}}^{n}, \cdots, v_{\Omega_{k}}^{n}, \cdots, u_{\Omega_{p}}^{n}\right)=0 .
$$

Now the question is whether the locally updated solution $\left(u_{\Omega_{1}}^{n}, \cdots, v_{\Omega_{k}}^{n}, \cdots, u_{\Omega_{p}}^{n}\right)$ is better than the non-updated solution $\left(u_{\Omega_{1}}^{n}, \cdots, u_{\Omega_{k}}^{n}, \cdots, u_{\Omega_{p}}^{n}\right)$ according to the overall residual function as defined in (3).

This algorithm can be extended for other multi-physics problems, such as the semiconductor device simulation problem in [11, the fluid-structure interaction problem in 2], and the magnetic reconnection problem in [13. However, we'll focus on the incompressible Navier-Stokes equations in this paper for now. And the rest of the paper is organized as follows. In Section 2, we introduce two parallel inexact Newton's methods with subspace correction. These algorithms are applicable for general nonlinear problems with local high nonlinearities. In Section 3, we provide some numerical results for solving the incompressible Navier-Stokes equations.

\section{Subdomain Jacobi-Newton Methods}

Suppose $\tilde{u}^{n}$ is the current approximate solution from the global Newton's method. For many PDE problems with local high nonlinearities, the surface plot of $F\left(\tilde{u}^{n}\right)$ would show a peak, say in $\Omega_{k}$, which is way higher than its neighboring regions. Many more Newton iterations are needed to remove this peak. During these Newton iterations, the components of $\tilde{u}^{n}$ on subdomains $\Omega_{1}, \cdots, \Omega_{k-1}, \Omega_{k+1}, \cdots, \Omega_{p}$ do not change much at all. In other words, the calculation on these subdomains is just a waste of time.

In order to isolate and to remove the peak, it seems to be a good idea not to use the original system (4), but to use the following nonlinear system of equations

$$
G^{n}(u)=\left(G_{1}^{n}(u), \cdots, G_{p}^{n}(u)\right)^{T}=0
$$

defined as:

$$
\left\{\begin{array}{l}
G_{k}^{n}(u)=F_{k}(u)=0 \\
G_{i}^{n}(u)=F_{i}(u)-F_{i}\left(\tilde{u}^{n}\right)=0, i \neq k .
\end{array}\right.
$$

We can clearly see that $G^{n}(u)=0$ amounts to smoothing the worst nonlinearity in the $k^{\text {th }}$ subdomain while maintaining others. That is, this approach eliminates the worst nonlinearity without over-correcting the rest of the components. 
The only issue is that the new nonlinear system (9) is as expensive to solve as the original system (4). In the rest of the paper, we propose two approximations to (9). Based on these approximate peak removing algorithms, we introduce the so-called Subdomain Jacobi-Newton (SJN) methods.

\section{Algorithm 1 (Subdomain Jacobi-Newton Framework). For $n=1,2, \cdots$,}

1. Perform the classical Newton iteration and generate a tentative iterate $\tilde{u}^{n}$.

2. Convergence test. If $\left\|F\left(\tilde{u}^{n}\right)\right\| \leq \epsilon_{\mathrm{r}}\left\|F\left(u^{0}\right)\right\|$ or $\left\|F\left(\tilde{u}^{n}\right)\right\| \leq \epsilon_{\mathrm{a}}$, a solution is found, or else go to next step. Here, $\epsilon_{\mathrm{r}}$ and $\epsilon_{\mathrm{a}}$ represent the relative and absolute machine epsilons, respectively, for the computation.

3. Inner iteration.

(a) Peak-finding. Find $k$ such that $\left\|F_{k}\left(\tilde{u}^{n}\right)\right\|_{2} \geq\left\|F_{i}\left(\tilde{u}^{n}\right)\right\|_{2}, \forall i \neq k$ and $\left\|F_{k}\left(\tilde{u}^{n}\right)\right\|_{2} \geq \rho\left\|F\left(\tilde{u}^{n}\right)\right\|_{2}$ where $0<\rho<1$. If such a $k$ does not exist, set $u^{n}=\tilde{u}^{n}$ and go back to step (1).

(b) Peak-removing. Form a new system of equations; solve it by one of the Jacobi methods using $\tilde{u}^{n}$ as the initial guess; call the solution $v^{n}$.

(c) Substitution. If $\left\|F\left(v^{n}\right)\right\|_{2}<\left\|F\left(\tilde{u}^{n}\right)\right\|_{2}, u^{n}=v^{n}$. Or else $u^{n}=\tilde{u}^{n}$. Go back to step (1).

One can adjust parameter $\rho$ so that only truly unbalanced nonlinearity in the context of the problem under consideration will be treated by this process. Next, we'll introduce two Jacobi methods to be used with the above algorithm.

\subsection{A Zeroth-Order Jacobi Method}

The standard nonlinear Jacobi method on one of the subdomains can be described using the global Newton's method on the following system:

$$
\left\{\begin{array}{l}
H_{k}^{n}(u)=F_{k}(u)=0, \\
H_{i}^{n}(u)=u_{\Omega_{i}}-\tilde{u}_{\Omega_{i}}^{n}=0, i \neq k,
\end{array}\right.
$$

i.e., it approximates the equation $G_{i}^{n}(u)=F_{i}(u)-F_{i}\left(\tilde{u}^{n}\right)=0$ for $i \neq k$, with

$$
H_{i}^{n}(u)=u_{\Omega_{i}}-\tilde{u}_{\Omega_{i}}^{n}=0 .
$$

That is, it uses piecewise constant to approximate the solution for $i \neq k$, where $k$ is the index of a subdomain where $u_{\Omega_{k}}$ is considered 'bad', e.g., having the largest residual $\left\|F_{k}\right\|_{2}$.

An advantage of this approach is, except on $\Omega_{k}$, that all equations are trivial; i.e., no calculation is necessary to obtain the solution.

\subsection{A First-Order Jacobi Method}

To remedy the crude approximation used in the zeroth-order Jacobi method, we form a new system of equations, 


$$
\begin{cases}H_{k}^{n}(u)=F_{k}(u) & =0, \\ H_{i}^{n}(u)=\frac{\partial F_{i}\left(\tilde{u}^{n}\right)}{\partial u}\left(u-\tilde{u}^{n}\right)=J_{i}\left(\tilde{u}^{n}\right)\left(u-\tilde{u}^{n}\right) & =0, i \neq k,\end{cases}
$$

i.e., it approximates the equation $G_{i}^{n}(u)=F_{i}(u)-F_{i}\left(\tilde{u}^{n}\right)=0$ for $i \neq k$, with

$$
H_{i}^{n}(u)=\frac{\partial F_{i}\left(\tilde{u}^{n}\right)}{\partial u}\left(u-\tilde{u}^{n}\right)=J_{i}\left(\tilde{u}^{n}\right)\left(u-\tilde{u}^{n}\right)=0 .
$$

In other words, it approximates the difference in $F_{i}$ at $\tilde{u}^{n}$ using its differential at $\tilde{u}^{n}$.

We remark here that the algorithms just proposed are similar in spirit to the recently introduced nonlinear preconditioning algorithms [459, but the nonlinear treatment here is more local.

\section{Numerical Experiments}

To demonstrate the efficiency and effectiveness of the new algorithms, we present some numerical results for solving the incompressible Navier-Stokes equations with

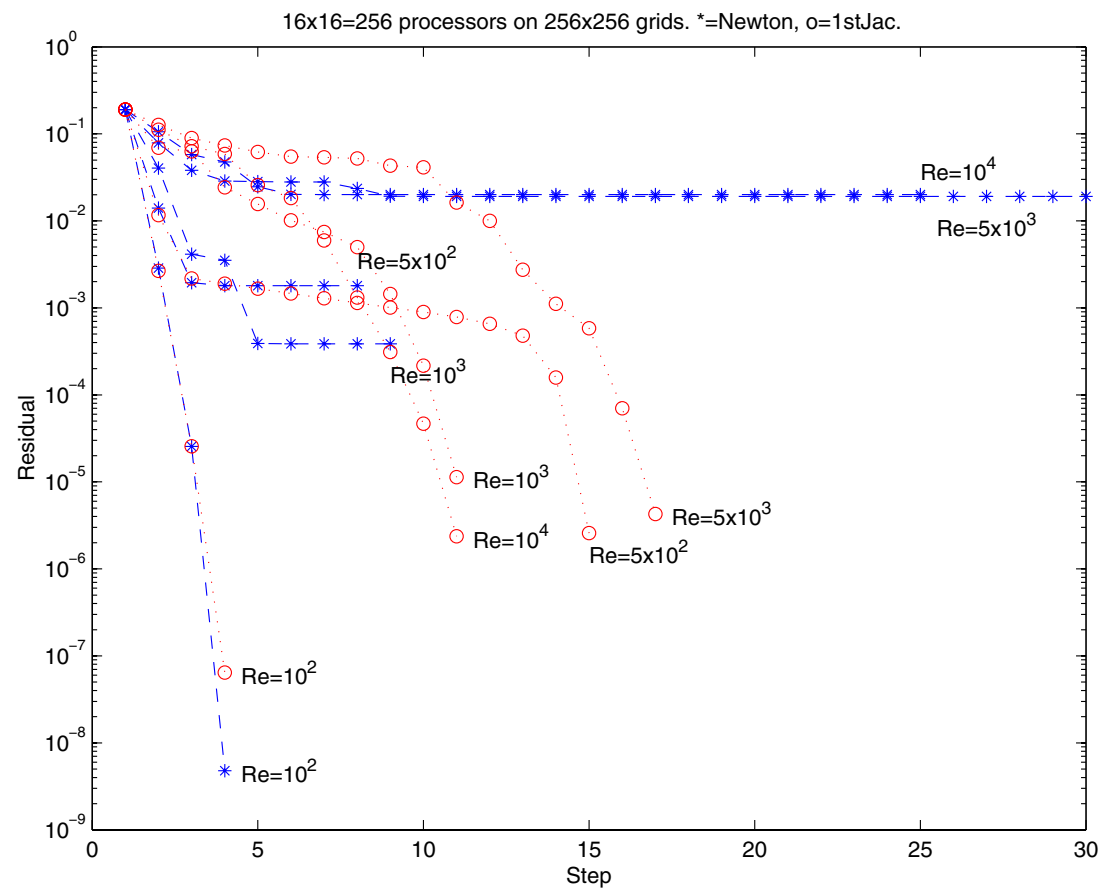

Fig. 1. Nonlinear residual history with varying Reynolds numbers. The classical inexact Newton is marked with $*$ and the new algorithm is marked with $\circ$. 
Residual of $\omega$-component after 1 iteration before subdomain iteration. $\mathrm{Re}=10^{3}$.

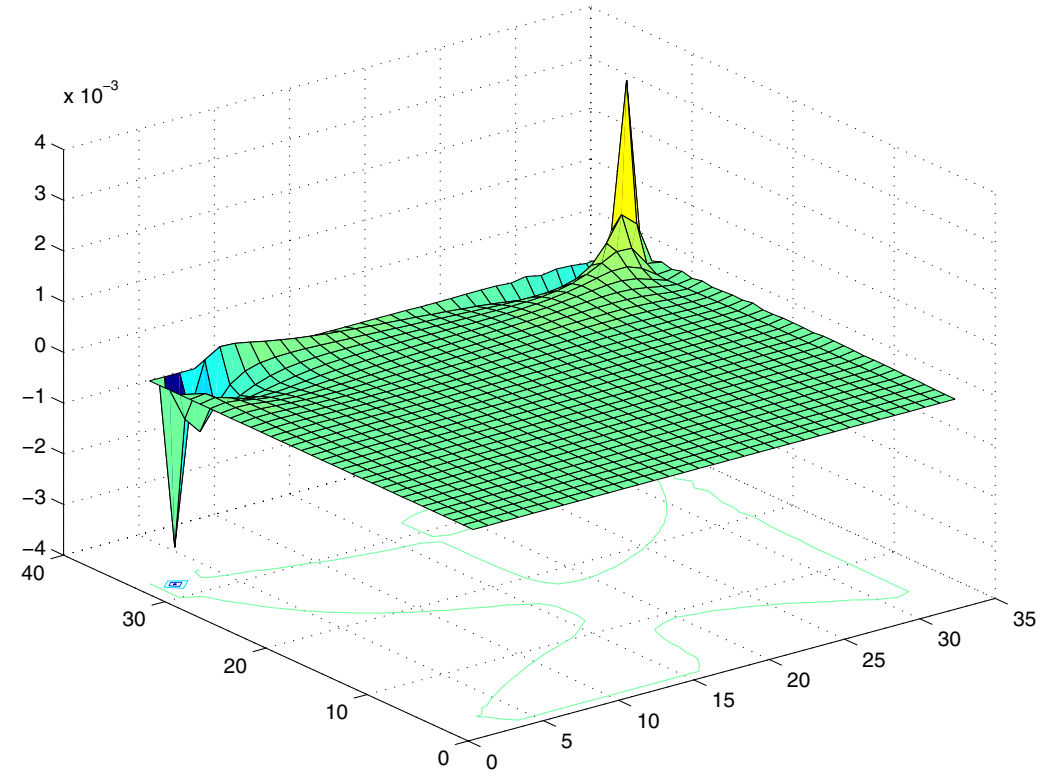

Residual of $\omega$-component after 1 iteration after subdomain iteration. $\mathrm{Re}=10^{3}$.

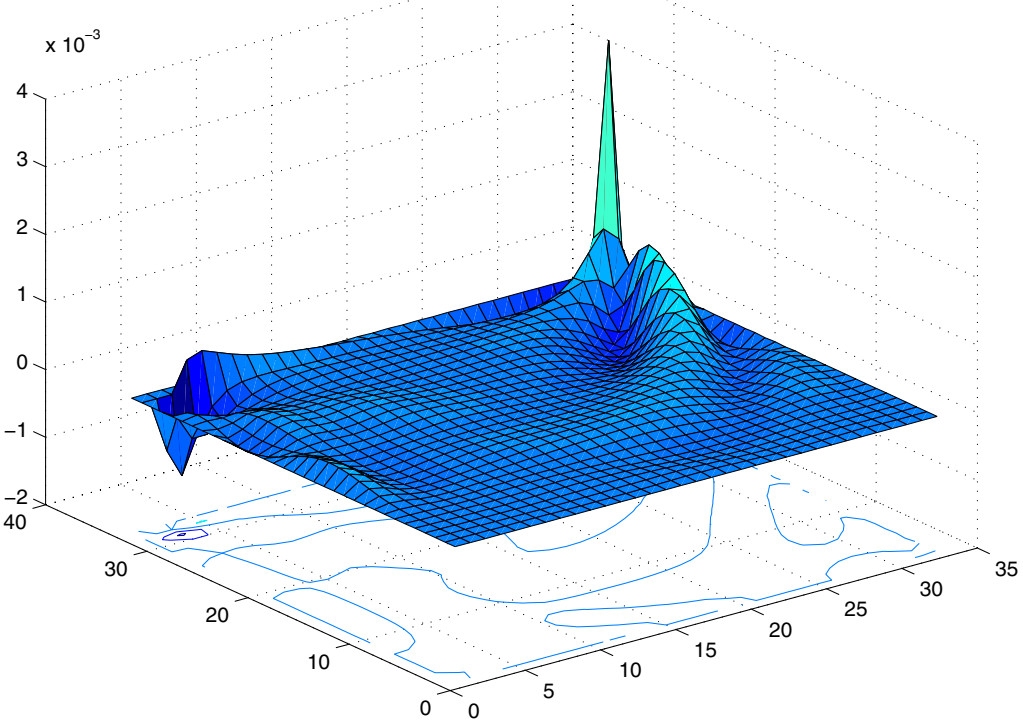

Fig. 2. The effect of subdomain Jacobi iterations: before (top) and after (bottom). One of the corner singularities is chosen to be eliminated by the inner Jacobi iterations. 
different Reynolds numbers. The implementation of the proposed algorithms is done using PETSc [1, and the results are obtained on an IBM BG/L. Both the zeroth order and the first order algorithms are implemented, but the first order algorithm is consistently better than the zeroth order algorithm, therefore, we will only report some preliminary experimental results of the first order algorithm.

The results are obtained for solving (11) on a $256 \times 256$ mesh with 256 processors. In all tests, the initial iterate is zero for $u, v$ and $\omega$. We stop the global and local nonlinear iteration if

$$
\left\|F\left(u^{n}\right)\right\| \leq \epsilon_{\mathrm{r}}\left\|F\left(u^{0}\right)\right\|
$$

where $\epsilon_{\mathrm{r}}=10^{-6}$ and $10^{-5}$ are used for the global Newton iterations and the SJN iterations, respectively. The linear iteration for solving the global Jacobian system is stopped if the relative tolerance

$$
\left\|F\left(u^{n}\right)-F^{\prime}\left(u^{n}\right) M_{n}^{-1}\left(M_{n} p^{n}\right)\right\| \leq \eta_{\mathrm{r}}\left\|F\left(u^{n}\right)\right\|
$$

is satisfied with $\eta_{\mathrm{r}}=10^{-4}$. The absolute tolerances for all iterations are $10^{-10}$. Here $M_{n}^{-1}$ is an additive Schwarz preconditioner [14] constructed using the Jacobian matrix $F^{\prime}$, and a partition of computational domain into 256 subdomains in a checker board fashion. The overlapping size is set to 2 , and the subdomain linear systems are solved by LU factorization.

In Fig. 1, we show the history of the norm of the residual of several test runs with different Reynolds numbers using the classical inexact Newton's method [3] (marked with "*") and the new algorithm (marked with "o"). As the Reynolds number increases, the nonlinear system becomes more difficult to solve. In fact, the classical method with the standard line search fails to converge once the Reynolds number exceeds $10^{2}$. We did not try to employ other techniques, such as pseudo-time stepping [10] or parameter/mesh continuations [1516, to improve the convergence of the classical method. On the other hand, SJN converges for a much larger range of Reynolds numbers as shown in Fig. 1 without employing any of the special tricks. In Fig. 2, we show an example of the surface plots of the residual function corresponding to the vorticity of the flow. The top picture is before a local high nonlinearity is removed, and the bottom picture shows the plot after the local Jacobi solver is applied to partially remove the local high nonlinearity.

\section{References}

1. Balay, S., Buschelman, K., Gropp, W., Kaushik, D., Knepley, M., McInnes, L., Smith, B., Zhang, H.: PETSc Users Manual, Argonne National Laboratory (2008)

2. Barker, A., Cai, X.-C.: NKS for fully coupled fluid-structure interaction with application. Lecture Notes in Computational Science and Engineering. Springer, Heidelberg (2009)

3. Cai, X.-C., Gropp, W.D., Keyes, D.E., Melvin, R.G., Young, D.P.: Parallel NewtonKrylov-Schwarz algorithms for the transonic full potential equation. SIAM J. Sci. Comput. 19, 246-265 (1998) 
4. Cai, X.-C., Keyes, D.E.: Nonlinearly preconditioned inexact Newton algorithm. SIAM J. Sci. Comput. 24, 183-200 (2002)

5. Cai, X.-C., Li, X.: Inexact Newton's methods with nonlinear restricted additive Schwarz preconditioning for problems with high local nonlinearities (in preparation)

6. Dennis Jr., J.E., Schnabel, R.B.: Numerical Methods for Unconstrained Optimization and Nonlinear Equations. SIAM, Philadelphia (1996)

7. Eisenstat, S.C., Walker, H.F.: Globally convergent inexact Newton methods. SIAM J. Optimization 4, 393-422 (1994)

8. Hirsch, C.: Numerical Computation of Internal and External Flows. John Wiley \& Sons, New York (1990)

9. Hwang, F.-N., Cai, X.-C.: A parallel nonlinear additive Schwarz preconditioned inexact Newton algorithm for incompressible Navier-Stokes equations. J. Comput. Phys. 204, 666-691 (2005)

10. Kelley, C.T., Keyes, D.E.: Convergence analysis of pseudo-transient continuation. SIAM J. Num. Anal. 35, 508-523 (1998)

11. Lanzkron, P.J., Rose, D.J., Wilkes, J.T.: An analysis of approximate nonlinear elimination. SIAM J. Sci. Comput. 17, 538-559 (1996)

12. Ortega, J., Rheinboldt, W.: Iterative Solution of Nonlinear Equations in Several Variables. SIAM, Philadelphia (2000)

13. Ovtchinnikov, S., Dobrian, F., Cai, X.-C., Keyes, D.: Additive Schwarz-based fully coupled implicit methods for resistive Hall magnetohydrodynamic problems. J. Comput. Phys. 225, 1919-1936 (2007)

14. Smith, B.F., Bjørstad, P.E., Gropp, W.D.: Domain Decomposition: Parallel Multilevel Methods for Elliptic Partial Differential Equations. Cambridge University Press, Cambridge (1996)

15. Young, D.P., Melvin, R.G., Bieterman, M.B., Johnson, F.T., Samant, S.S.: Global convergence of inexact Newton methods for transonic flow. Int. J. Numer. Meths. Fluids 11, 1075-1095 (1990)

16. Young, D.P., Mervin, R.G., Bieterman, M.B., Johnson, F.T., Samant, S.S., Bussoletti, J.E.: A locally refined rectangular grid finite element method: Application to computational fluid dynamics and computational physics. J. Comput. Phys. 92, 1-66 (1991) 